\title{
Heterogeneous Oblate Primaries in Photo-gravitational CR5BP with Kite Configuration
}

\author{
Abdullah A. Ansari ${ }^{1,3}$, Prashant Kumar ${ }^{2,3}$, Mehtab Alam ${ }^{3}$ \\ 1 Department of Mathematics, College of Science Al-Zulfi, Majmaah University, KSA \\ 2 Division of Mathematics, School of Basic and Applied Sciences, \\ Galgotias University, Greater Noida, U. P., India. \\ 3 International Center for Advanced Interdisciplinary Research (ICAIR) \\ Ratiya Marg, Sangam Vihar, New Delhi, India 110062 \\ Correspondence to: Abdullah A. Ansari, Email: a.ansari@mu.edu.sa
}

\begin{abstract}
This paper presents the investigation of the motion of infinitesimal body in the circular restricted five-body problem in which four bodies are taken as heterogeneous oblate spheroid with different densities in three layers and sources of radiation pressure. These four primaries are moving on the circumference of a circle and form a kite configuration. After evaluating the equations of motion and Jacobi-integral, we study the numerical part of the paper such as equilibria, zero-velocity curves and regions of motion. Finally, we examine the stability of the equilibria and observed that all the equilibria are unstable.
\end{abstract}

Keywords: Heterogeneous oblate spheroid, CR5BP, Kite configuration, Equilibria, Zero-velocity curves, Stability

\section{Introduction}

The restricted problem is one of the attracting and interesting problem in the field of mechanics and astronomy due to its applications. The history of restricted problem starts before four centuries with Euler and Lagranges in 1772. The restricted problem studied in three-body, four-body and five-body by many researchers with many perturbations as the different shapes of the primaries (oblate, triaxial, heterogeneous, finite straight segments etc.), solar radiation pressure, albedo effect, yarkovsky effects, drags, resonances, Coriolis and centrifugal forces, variable mass, etc. In the restricted three-body problem (R3BP), the two bodies, known as primaries, are moving in circular orbits around their common center of mass and third body, known as infinitesimal body, are moving in space under the influence of these primaries but not influencing them. Many mathematicians have studied this problem with many perturbations as: Radzievskii [15, 16], Chernikov 9, Bhatnagar and Hallan 8, Singh and Lake [21, Suraj et al. 22, 23], Abouelmagd and Mostafa 2], Shalini et al. [19, 20, Ansari et al. [5], etc.

In the restricted four-body problem (R4BP), three-bodies are forming either Eulerian configuration or Lagrangian configuration. And the fourth body is moving under the influence of these four primaries but not affecting them. Many researchers have investigated this problem as: Kalvouridis et al. [10, Papadakis [14, Abdullah et al. [1], Baltagianis [7, Kumari and Kushvah [11, Ansari et al. [3, 4, etc.

On the other hand, in the restricted five-body problem (R5BP), four primaries are moving in their mutual gravitational forces and the fifth body is moving under the influence of them but not influencing them. Many scientists have illustrated this problem as: Ollongren [13, Marchesin et al. [12, Shahbaz et al. [18, Shoaib et al. [17, Zotos et al. 24, Ansari et al. 6].

This paper is organized as follows: In the second section, we have determined the equations of motion and Jacobian integral. In the third section, we have done the numerical works. In the fourth section, we have examined the stability of the equilibria. And finally, we have concluded the problem.

\section{Description of the Problem and Equations of Motion}

Here, we have considered the restricted five-body problem with circular kite configuration. In which four bodies, taken as heterogeneous oblate spheroid with separate densities of three layers and also sources of radiation, are placed at the circumference of the circle with radius $R$ and also these four primaries are forming circular kite $\left(P_{1} P_{2} P_{3} P_{4}\right)$ which has two triangles. $P_{1} P_{2} P_{3}$ is an equilateral triangle with side $\ell$, 


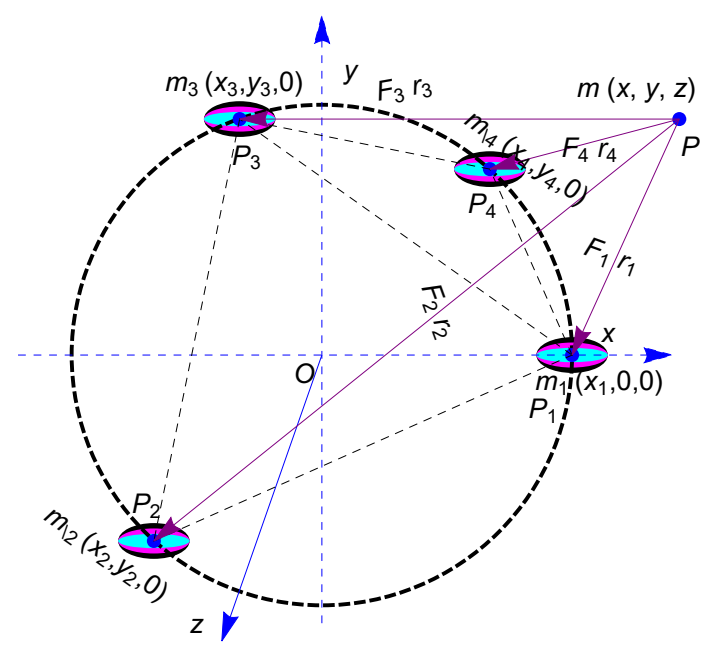

Figure 1: Heterogeneous oblate primaries in circular kite configuration.

while $P_{1} P_{3} P_{4}$ is an isosceles triangle. The relation between $\ell$ and $R$ is $\ell=\sqrt{3} R$. The fifth body having negligible mass as compare to the primaries and known as infinitesimal body, is moving in the space under the influence of these four primaries but not influencing them. Let the principal axes of the heterogeneous oblate spheroid remain parallel to the synodic axes $O x y z$ in the complete motion and revolving with angular velocity $\vec{\omega}=n \widehat{k}$ about z-axis. Where the center of the circle is $O$ which is also taken as origin and center of mass of the primaries. Let $m_{1}, m_{2}, m_{3}, m_{4}$ and $m$ be the masses of the primaries with $m_{1}=m_{3}$ (in all aspects) and infinitesimal body and also let the coordinates of the vertices of kite $P_{1}, P_{2}, P_{3}, P_{4}$ and infinitesimal body $P$ be $\left(x_{1}, 0,0\right)=(R, 0,0),\left(x_{2}, y_{2}, 0\right)=\left(-\frac{R}{2},-\frac{\sqrt{3} R}{2}, 0\right),\left(x_{3}, y_{3}, 0\right)=\left(-\frac{R}{2}, \frac{\sqrt{3} R}{2}, 0\right)$, $\left(x_{4}, y_{4}, 0\right)=\left(\frac{R}{2}, \frac{\sqrt{3} R}{2}, 0\right)$ and $(x, y, z)$ respectively.

The total force acting on the infinitesimal body is

$$
\vec{F}=\left(1-\alpha_{1}\right) \overrightarrow{F_{1}}+\left(1-\alpha_{2}\right) \overrightarrow{F_{2}}+\left(1-\alpha_{3}\right) \overrightarrow{F_{3}}+\left(1-\alpha_{4}\right) \overrightarrow{F_{4}}
$$

i.e., $\overrightarrow{F_{p}}$ and $\alpha_{p}$ are the force and solar radiation pressure factor due to corresponding primaries $(\mathrm{p}=1,2$, $3,4)$.

If $\vec{r}=x \vec{i}+y \vec{j}+z \vec{k}$, is the position vector of the infinitesimal body then the equation of motion of $\mathrm{m}$ in the vector form will be

$$
m\left(\frac{\partial^{2} \vec{r}}{\partial t^{2}}+2 \vec{\omega} \times \frac{\partial \vec{r}}{\partial t}+\frac{\partial \vec{\omega}}{\partial t} \times \vec{r}+\vec{\omega} \times(\vec{\omega} \times \vec{r})\right)=\vec{F} .
$$

where first, second, third and fourth terms of the equation (2) are relative acceleration, Coriolis acceleration, Euler's acceleration and centrifugal acceleration respectively.

The gravitational potential of the heterogeneous oblate spheroid with three layers of densities $\rho_{q}$ and axes $\left(a_{q}, b_{q}, c_{q}\right), \rho_{q}<\rho_{q+1}, a_{q}<a_{q+1}, b_{q}<b_{q+1}, c_{q}<c_{q+1}$ at any point is

$$
V=-\frac{\operatorname{mass} G}{R_{1}}-\frac{d G}{2 R_{1}^{3}}-\frac{3 d G z^{2}}{2 R_{1}^{5}}
$$

where, $R_{1}=$ Distance between heterogeneous oblate body and the point at which potential is going to be found and

$$
d=\frac{4 \pi}{3} \sum_{q=1}^{3}\left(\left(\rho_{q}-\rho_{q+1}\right) a_{q} b_{q} c_{q} \sigma_{q}\right), \sigma_{q}=\frac{\left(\left(a_{q}\right)^{2}-\left(c_{q}\right)^{2}\right)}{5}, \rho_{4}=0 . \quad \text { (Ansari [5] ) }
$$

Hence, the total potential at point $\mathrm{P}$ due to $m_{1}, m_{2}, m_{3}$ and $m_{4}$ is 


$$
V=\sum_{p=1}^{4}\left(-\frac{m_{p} G}{r_{p}}-\frac{d_{p} G}{2 r_{p}^{3}}-\frac{3 d_{p} G z^{2}}{2 r_{p}^{5}}\right)
$$

with,

$$
r_{p}^{2}=\left(x-x_{p}\right)^{2}+\left(y-y_{p}\right)^{2}+z^{2} .
$$

To fix the units, the sum of the masses $m_{1}+m_{2}+m_{3}+m_{4}=\mathrm{M}=1$, the radius of the circle is considered as unity i.e. $R=1$, and unit of time is chosen in such a way that $G=1$ and $n=1$. Let $\mu_{p}=\frac{m_{p}}{M}, \mu_{2}=\mu$ and $\mu_{4}=\alpha \mu, \Rightarrow \mu_{1}=\mu_{3}=\frac{1-\mu-\alpha \mu}{2}$.

Hence from equation (2), the dimensionless equations of motion in the cartesian form will be

$$
\left\{\begin{array}{c}
\ddot{x}-2 \dot{y}=\frac{\partial \Psi}{\partial x} \\
\ddot{y}+2 \dot{x}=\frac{\partial \Psi}{\partial y} \\
\ddot{z}=\frac{\partial \Psi}{\partial z}
\end{array}\right.
$$

where, $\frac{\partial \Psi}{\partial x}, \frac{\partial \Psi}{\partial y}$ and $\frac{\partial \Psi}{\partial z}$ are the partial derivatives of $\Psi$ w.r.to $\mathrm{x}, \mathrm{y}$ and $\mathrm{z}$ respectively, and

$$
\begin{gathered}
\Psi=\frac{1}{2}\left(x^{2}+y^{2}\right)+\sum_{p=1}^{4} \beta_{p}\left(\frac{\mu_{p}}{r_{p}}+\frac{d_{p}}{2 r_{p}^{3}}+\frac{3 d_{p} z^{2}}{2 r_{p}^{5}}\right), \\
\beta_{p}=1-\alpha_{p}, d_{p}=\frac{4 \pi}{3} \sum_{q=1}^{3}\left(\left(\delta_{q}^{p}-\delta_{q+1}^{p}\right)\left(A_{q}^{p}\right)^{2} C_{q}^{p} \sigma_{q}^{p}\right), \\
A_{q}^{p}=\frac{a_{q}^{p}}{R}, C_{q}^{p}=\frac{c_{q}^{p}}{R}, \sigma_{q}^{p}=\frac{\left(\left(a_{q}^{p}\right)^{2}-\left(c_{q}^{p}\right)^{2}\right)}{5 R^{2}} \cdot(p=1,2,3,4)
\end{gathered}
$$

After multiplying one by one in the system (6) with $\dot{x}, \dot{y}, \dot{z}$ respectively and adding, we get the JacobiIntegral as

$$
v^{2}=2 \Psi-C .
$$

where $v^{2}=\dot{x}^{2}+\dot{y}^{2}+\dot{z}^{2}$ is the velocity of the infinitesimal body and $C$ is the Jacobi-Integral constant which is conserved.

\section{Analysis of the Problem}

In this section, we have numerically investigated the equilibria, zero-velocity curves and regions of motion in three planes (x-y, x-z and y-z planes) and shown the dynamical behaviour of the infinitesimal body. (In the complete investigation, we have taken: $\mu=0.3, \alpha=0.01, d_{1}=d_{3}=9.83933 \times 10^{-18}, d_{2}=1.58302 \times 10^{-7}$, $d_{4}=3.13153 \times 10^{-8}, \beta_{1}=\beta_{2}=\beta_{3}=\beta_{4}=\beta$.) (Ansari [5])

\subsection{Evolution of equilibria}

To evaluate the equilibrium points, we have to put right hand sides of system (6) equal to zero. i.e.,

$$
\begin{aligned}
& \frac{\partial \Psi}{\partial x}=0, \\
& \frac{\partial \Psi}{\partial y}=0, \\
& \frac{\partial \Psi}{\partial z}=0 .
\end{aligned}
$$

After solving these equations by Mathematica software, we got figure 2, figure 3 and figure 4 in $x-y, x-z$ and $\mathrm{y}-\mathrm{z}$ plane respectively. 
Heterogeneous Oblate Primaries in Photo-gravitational CR5BP with Kite Configuration

\subsubsection{Equilibria in $x-y$ plane}

In this plane, we have plotted the equilibrium points at four different values of $\beta(0.5,0.8,0.9,1)$. At $\beta=0.5$ and 0.8 , we got four equilibrium points $\left(L_{1}, L_{2}, L_{3}, L_{4}\right)$ in figures $2 \mathrm{a}$ and $2 \mathrm{~b}$ respectively, while $\beta=0.9$ and 1 , we got one extra i.e. five equilibrium points $\left(L_{1}, L_{2}, L_{3}, L_{4}, L_{5}\right)$ in figures $2 \mathrm{c}$ and $2 \mathrm{e}$ respectively. Figures $2 \mathrm{~d}$ and $2 \mathrm{f}$ are the zoomed figures of the figures $2 \mathrm{c}$ and $2 \mathrm{e}$ respectively. Red stars are denoted as the locations of the photo-gravitational primaries $\left(m_{1}, m_{2}, m_{3}\right.$ and $\left.m_{4}\right)$. From all the figures, we observed that equilibrium points are near the primaries except one. i.e., $L_{1}$ is near $m_{1}, L_{3}$ is near $m_{3}$, $L_{4}$ is near $m_{2}, L_{5}$ is near $m_{4}$ while $L_{2}$ is near the origin. And also it is observed that as we increase the value of $\beta$ these equilibrium points start moving away.

Therefore, we can say that these shapes of the primaries as heterogeneous and radiation factor have great impact as we know in the classical case of restricted three-body problem, researchers got five equilibrium points where three are collinear and two are non-collinear.

\subsubsection{Equilibria in $\mathrm{x}-\mathrm{z}$ and $\mathrm{y}-\mathrm{z}$ planes}

Here, we got two equilibrium points $\left(L_{1}\right.$ and $\left.L_{2}\right)$ in $\mathrm{x}-\mathrm{z}$ plane (figure $3 \mathrm{a}$ ) and one equilibrium point $\left(L_{1}\right)$ in $y$-z plane (figure $4 \mathrm{a}$ ) for the same four values of $\beta$. Figures $3 \mathrm{~b}$ and $4 \mathrm{~b}$ are the zoomed parts of figures $3 \mathrm{a}$ and 4 a near $L_{1}$ respectively. In x-z plane, $L_{2}$ is near the origin while $L_{1}$ is right side of the origin and on the x-axis. And in $\mathrm{y}-\mathrm{z}$ plane, $L_{1}$ is near the origin. From both the figures we observed that, as we increase the values of $\beta$, the equilibrium points move away from the origin.

\subsection{Zero-velocity curves}

We have studied the zero-velocity curves of the infinitesimal body in three planes i.e., $x-y, x-z$ and $y-z$ planes. To draw the zero-velocity curves, first of all, we have to compute the values of Jacobi-constant $\mathrm{C}$ corresponding to each equilibrium points from equation (7). After computing the values of $\mathrm{C}$, we have drawn the zero-velocity curves for different values of the radiation factor $\beta$ (Figure 5).

Figure 5a shows four zero-velocity curves corresponding to each Jacobian constants $C_{L_{1}}, C_{L_{2}}, C_{L_{3}}$ and $C_{L_{4}}$ for radiation factor $\beta=0.5$. The curve due to $C_{L_{1}}$ (Black) covers $L_{2}, L_{4}, m_{2}$ and $m_{4}$ while $L_{1}$ and $L_{3}$ become limiting points. The curve due to $C_{L_{2}}$ (Blue) cover $L_{2}$ only. The curve due to $C_{L_{3}}$ (Red) covers all the primaries in the circular form. While the curve due to $C_{L_{4}}$ (Green) covers $L_{2}$ and $m_{4}$.

Figure 5b also shows four zero-velocity curves corresponding to $C_{L_{1}}, C_{L_{2}}, C_{L_{3}}$ and $C_{L_{4}}$ for radiation factor $\beta=0.8$. The curve due to $C_{L_{1}}, C_{L_{2}}$ and $C_{L_{3}}$ shows the same phenomenon as figure $5 \mathrm{a}$. But the curve due to $C_{L_{4}}$ (Green) covers $L_{2}, L_{4}, m_{2}$ and $m_{4}$.

Figures $5 \mathrm{c}$ and $5 \mathrm{~d}$ shows five zero-velocity curves corresponding to $C_{L_{1}}, C_{L_{2}}, C_{L_{3}}, C_{L_{4}}$ and $C_{L_{5}}$ for radiation factors $\beta=0.9$ and 1 respectively. The curves due to corresponding Jacobian constants shows the same phenomenon as the curves due to $C_{L_{1}}$ and $C_{L_{3}}$ cover all the primaries in circular form. The curve due to $C_{L_{2}}$ (Blue) covers $L_{2}$ only. The curve due to $C_{L_{4}}$ (Green) covers $L_{2} L_{4}, L_{5}, m_{2}$ and $m_{4}$. The curve due to $C_{L_{5}}$ (Purple) covers $L_{2}, L_{5}$ and $m_{4}$.

Again in $x-z$ and $y-z$ planes, we have shown the zero-velocity curves only at the value of radiation factor $\beta=0.9$. From figure $6 \mathrm{a}$, the curve due to $C_{L_{1}}$ (Black) covers $L_{1}$ and $L_{2}$ but it is not bounded. The curve due to $C_{L_{2}}$ covers only $L_{2}$ and not bounded. Then from figure $6 \mathrm{~b}$, the curve due to $C_{L_{1}}$ covers $L_{1}$ and not bounded.

\subsection{Regions of motion}

In this part, we have plotted the regions of motion to study the dynamical behaviour of infinitesimal body under the effect of four heterogeneous and source of radiation primaries in three planes ( $\mathrm{x}-\mathrm{y}$ (fig 7), $\mathrm{x}-\mathrm{z}$ (fig $8 \mathrm{a}, \mathrm{b})$ and $\mathrm{y}-\mathrm{z}$ planes (fig 8c)). The color regions represent the prohibited regions which varies according to the variation of the values of the Jacobian constants. The fifth body can move freely in the region other than the colored region. 

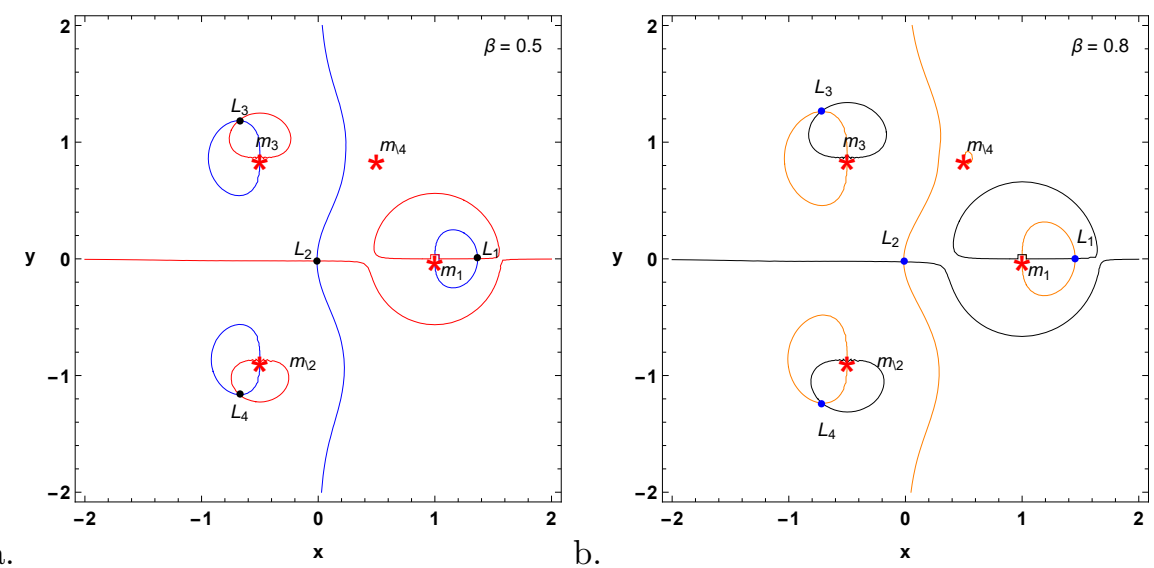

a.

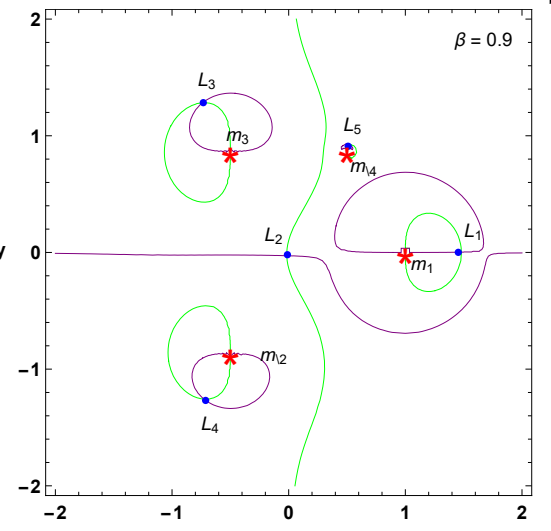

c.

c.

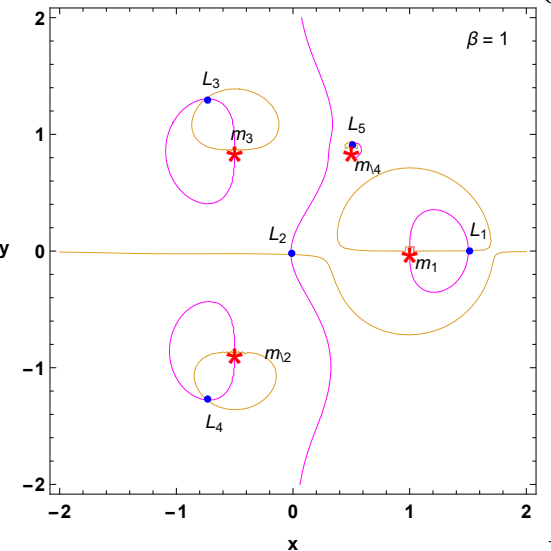

b.

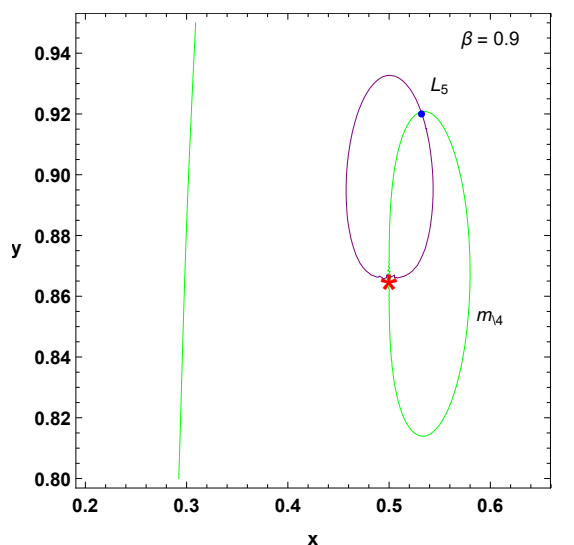

d.

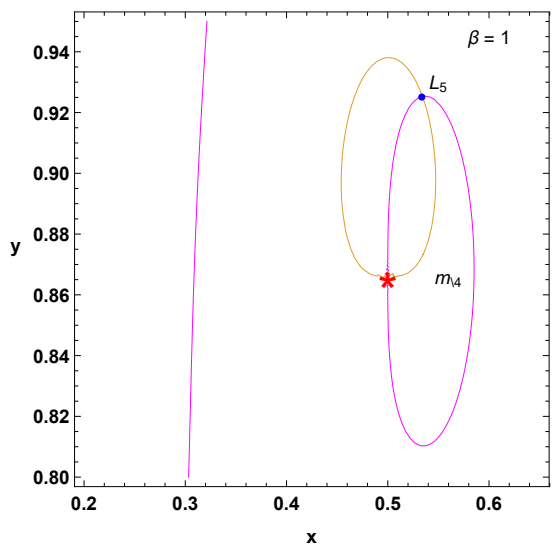

Figure 2: Locations of equilibrium points in $\mathrm{x}$-y-plane at different values of radiation factor $\beta$. a. at 0.5 , b. at 0.8 , c. at 0.9 , d. zoomed part of figure c near $L_{5}$, e. at 1 and f. zoomed part of figure e near $L_{5}$. 

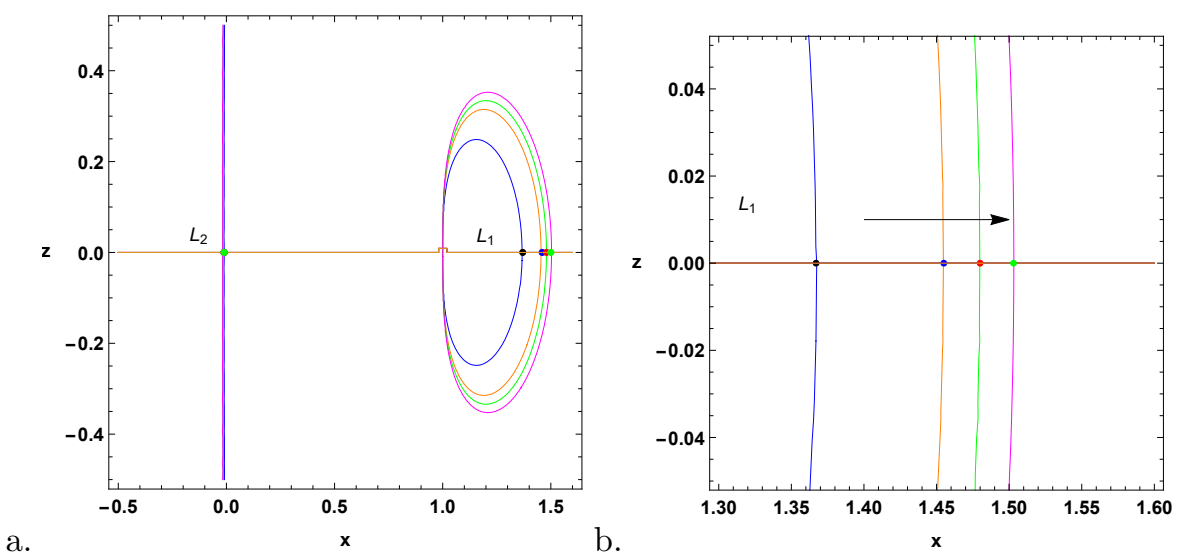

Figure 3: a. Locations of equilibrium points in $\mathrm{x}$-z-plane at different values of radiation factor $\beta$ at 0.5 (Black), at 0.8 (Blue), at 0.9(Red), at 1(Green), b. Zoomed part of the figure a near $L_{1}$.
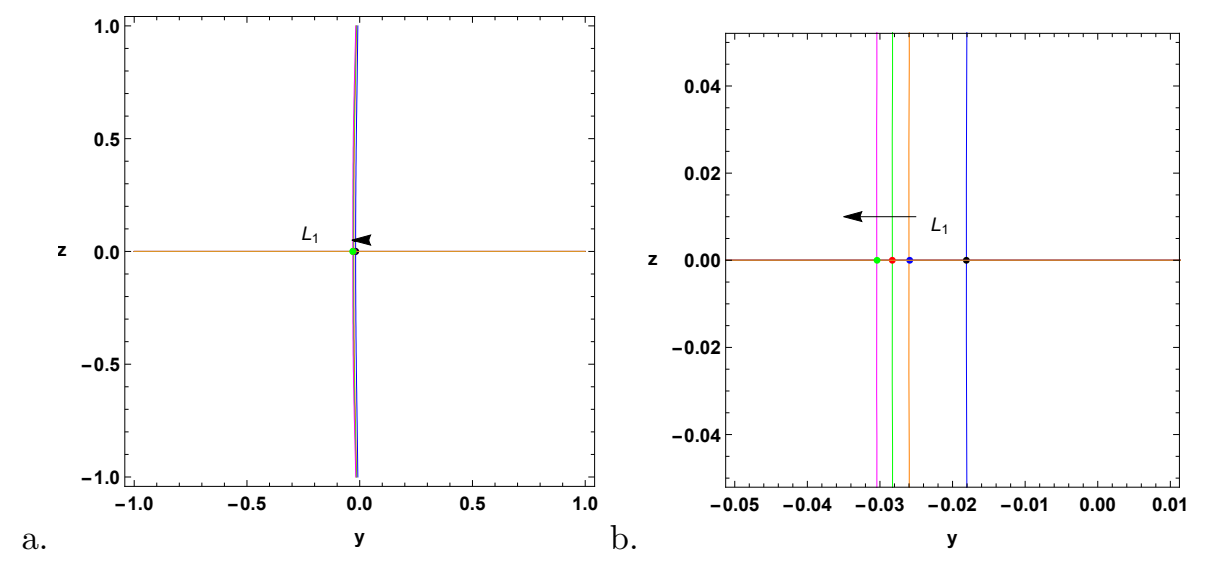

Figure 4: a. Locations of equilibrium points in y-z-plane at different values of radiation factor $\beta$ at 0.5 (Black), at 0.8 (Blue), at 0.9 (Red), at 1 (Green), b. Zoomed part of the figure a near $L_{1}$. 

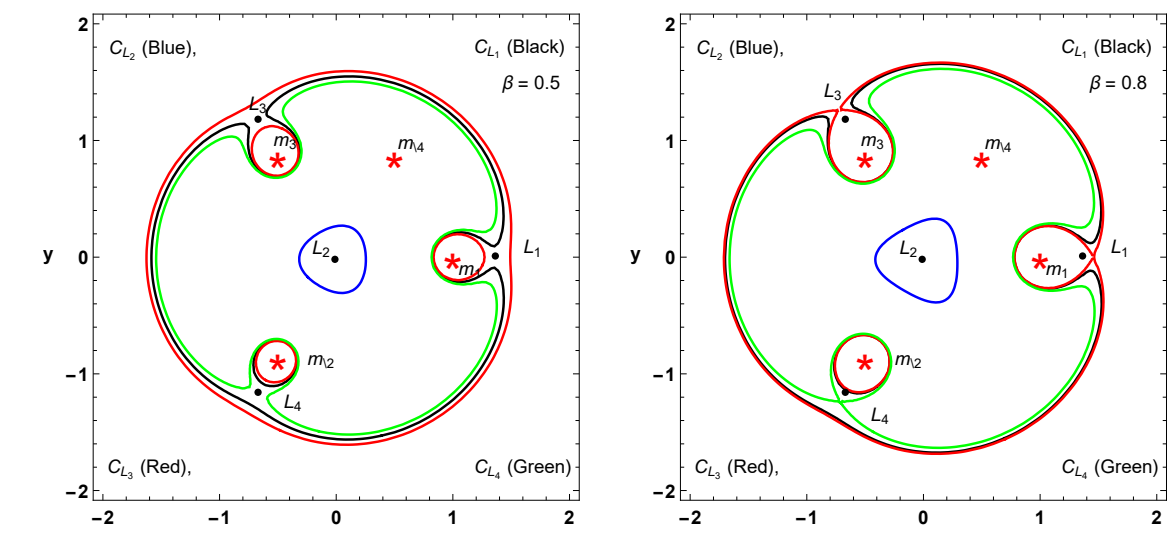

a.

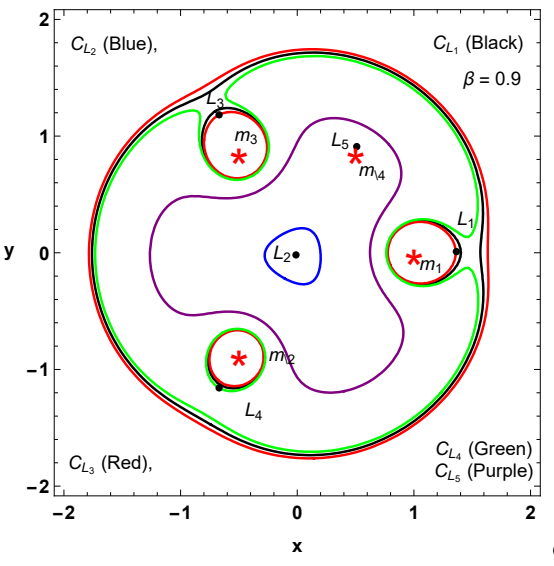

b.

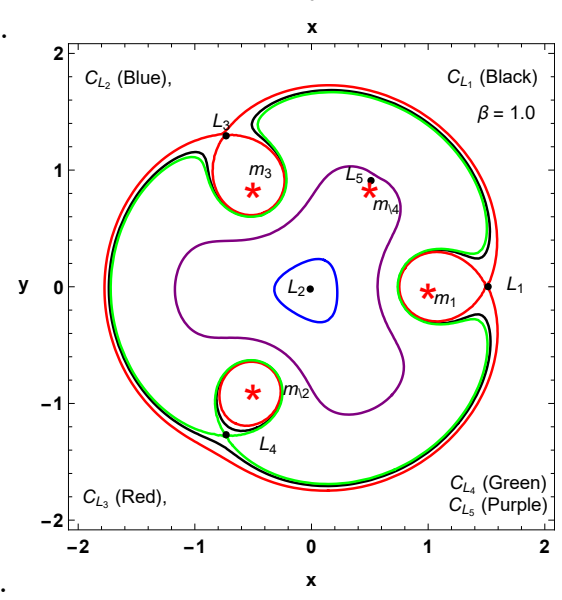

Figure 5: Zero-velocity curves in $\mathrm{x}$-y-plane at different values of the radiation factor $\beta$. a. 0.5, b. 0.8 , c. 0.9 , d. 1.

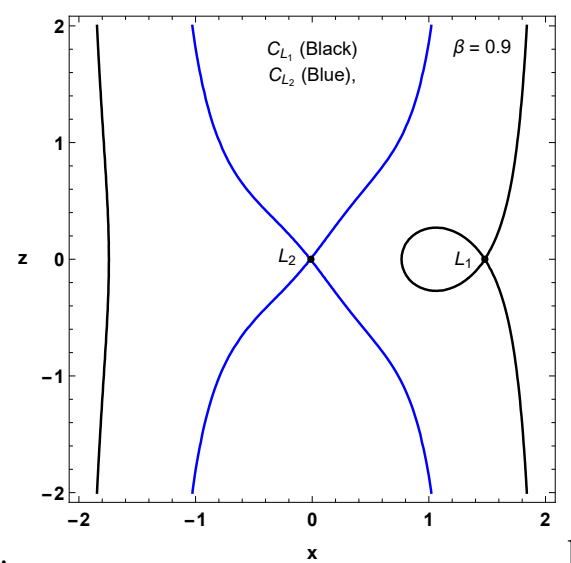

a.

Figure 6: Zero-velocity curves at the radiation factor $\beta=0.9$. a. in $\mathrm{x}$-z-plane and $\mathrm{b}$. in $\mathrm{y}$-z-plane. 

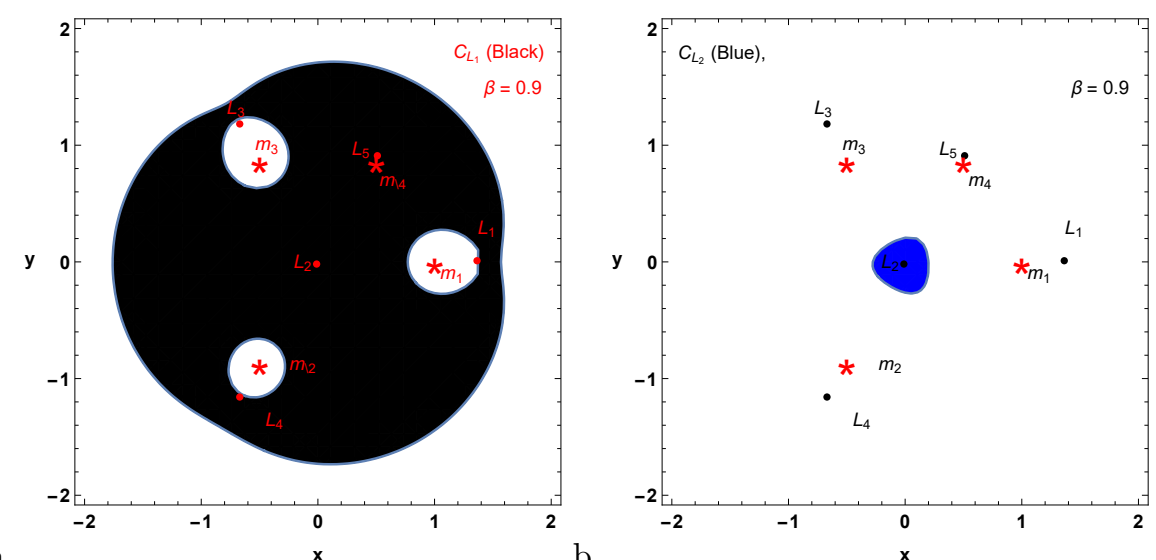

a.

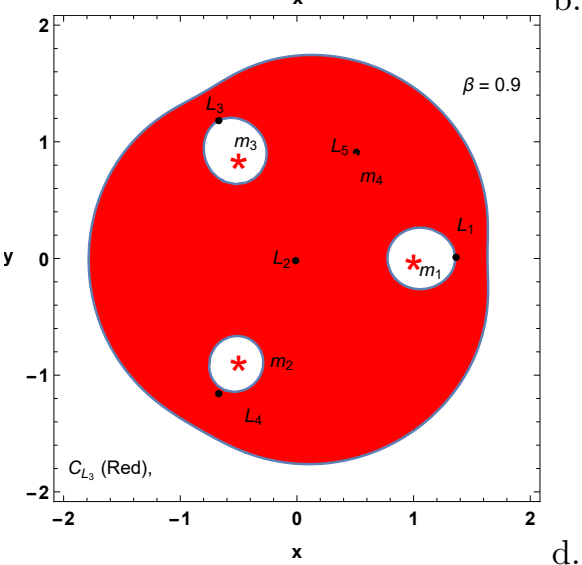

b.

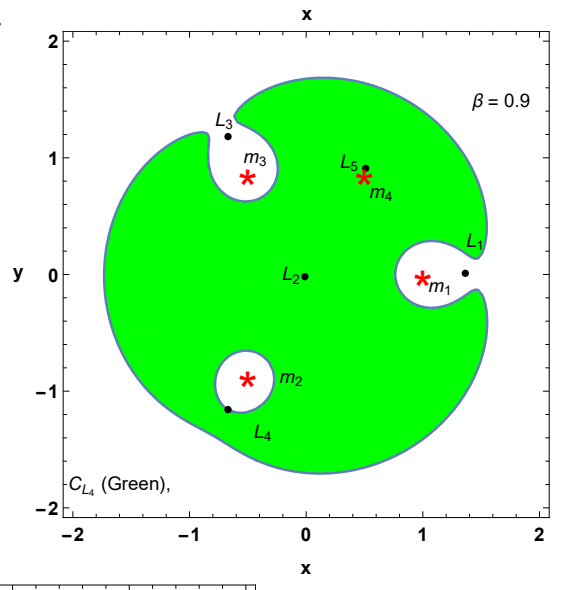

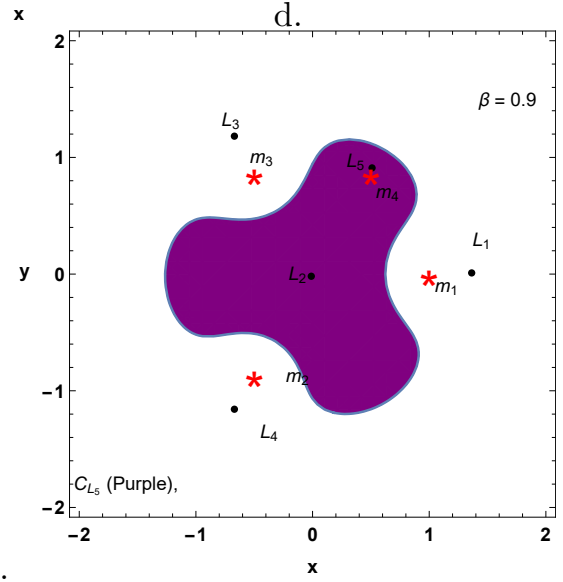

Figure 7: Regions of motion in x-y-plane at the radiation factor $\beta=0.9$ and figures corresponds to a. $C_{L_{1}}$, b. $C_{L_{2}}$, c. $C_{L_{3}}$, d. $C_{L_{4}}$, e. $C_{L_{5}}$. 


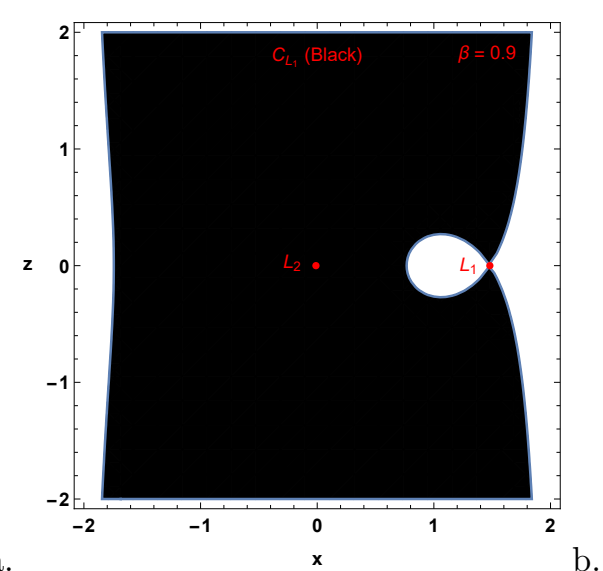

a.
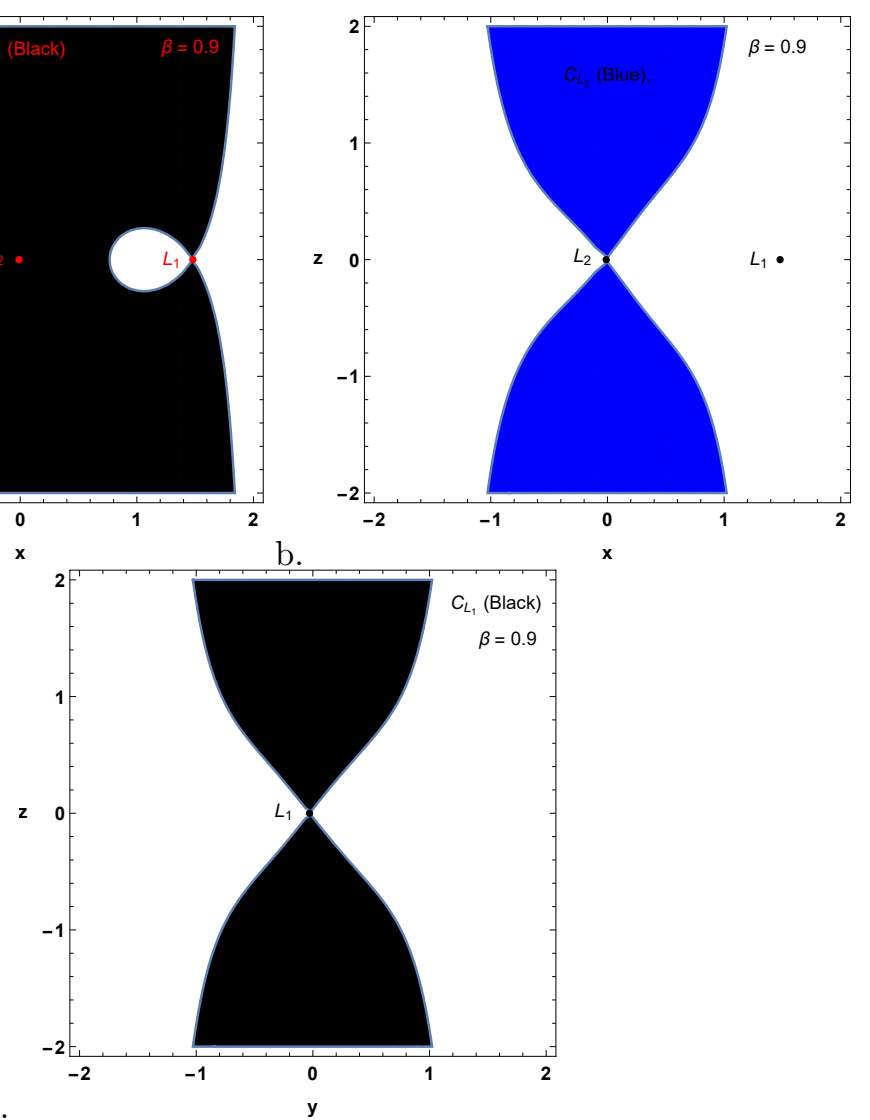

Figure 8: Regions of motion at the radiation factor $\beta=0.9$. a, b. in x-z-plane and c. in y-z-plane at the corresponding values of the Jacobian constant. 


\section{Stability of Equilibria}

In this section, we have examined the stability of equilibria in the motion. The stability of motion means bounded displacements and velocities which are bounded functions of time in the neighbourhood of the equilibrium points. Let the coordinates of any one of the equilibrium points be denoted by $\left(x_{0}, y_{0}, z_{0}\right)$ and $\left(x_{0}^{1}, y_{0}^{1}, z_{0}^{1}\right)$ denote small displacements of the infinitesimal particle from the equilibrium points. i.e., $x=x_{0}+x_{0}^{1}, y=y_{0}+y_{0}^{1}, z=z_{0}+z_{0}^{1}$.

We assume that the displacements are sufficiently very small that the Taylor expansions of $\psi_{x}, \psi_{y}, \psi_{z}$, in the region surrounding the equilibrium points can be written as

$$
\left\{\begin{array}{l}
\psi_{x}=x_{0}^{1}\left(\psi_{x x}\right)^{0}+y_{0}^{1}\left(\psi_{x y}\right)^{0}+z_{0}^{1}\left(\psi_{x z}\right)^{0} \\
\psi_{y}=x_{0}^{1}\left(\psi_{y x}\right)^{0}+y_{0}^{1}\left(\psi_{y y}\right)^{0}+z_{0}^{1}\left(\psi_{y z}\right)^{0} \\
\psi_{z}=x_{0}^{1}\left(\psi_{z x}\right)^{0}+y_{0}^{1}\left(\psi_{z y}\right)^{0}+z_{0}^{1}\left(\psi_{z z}\right)^{0}
\end{array}\right.
$$

where the partial derivatives are evaluated at the point $\left(x_{0}, y_{0}, z_{0}\right)$.

Therefore, the system (6) can be written as,

$$
\left\{\begin{array}{c}
\ddot{x}_{0}^{1}-2 \dot{y}_{0}^{1}=x_{0}^{1}\left(\psi_{x x}\right)^{0}+y_{0}^{1}\left(\psi_{x y}\right)^{0}+z_{0}^{1}\left(\psi_{x z}\right)^{0} \\
\ddot{y}_{0}^{1}+2 \dot{x}_{0}^{1}=x_{0}^{1}\left(\psi_{y x}\right)^{0}+y_{0}^{1}\left(\psi_{y y}\right)^{0}+z_{0}^{1}\left(\psi_{y z}\right)^{0} \\
\ddot{z}_{0}^{1}=x_{0}^{1}\left(\psi_{z x}\right)^{0}+y_{0}^{1}\left(\psi_{z y}\right)^{0}+z_{0}^{1}\left(\psi_{z z}\right)^{0} .
\end{array}\right.
$$

To solve the above equations, let

$$
x_{0}^{1}=A e^{\lambda t}, y_{0}^{1}=B e^{\lambda t}, z_{0}^{1}=C e^{\lambda t},
$$

where A, B, C and $\lambda$ are parameters. Substituting equation (13) in equation (12), and the rearrangement yield

$$
\left\{\begin{array}{c}
A\left(\lambda^{2}-\left(\psi_{x x}\right)^{0}\right)+B\left(-2 \lambda-\left(\psi_{x y}\right)^{0}\right)-C\left(\psi_{x z}\right)^{0}=0 \\
A\left(2 \lambda-\left(\psi_{y x}\right)^{0}\right)+B\left(\lambda^{2}-\left(\psi_{y y}\right)^{0}\right)-C\left(\psi_{y z}\right)^{0}=0 \\
-A\left(\psi_{z x}\right)^{0}-B\left(\psi_{z y}\right)^{0}+C\left(\lambda^{2}-\left(\psi_{z z}\right)^{0}\right)=0
\end{array}\right.
$$

This will have a non-trivial solution for $\mathrm{A}, \mathrm{B}$ and $\mathrm{C}$, if

$$
\left|\begin{array}{ccc}
\left(\lambda^{2}-\left(\psi_{x x}\right)^{0}\right) & \left(-2 \lambda-\left(\psi_{x y}\right)^{0}\right) & -\left(\psi_{x z}\right)^{0} \\
\left(2 \lambda-\left(\psi_{y x}\right)^{0}\right) & \left(\lambda^{2}-\left(\psi_{y y}\right)^{0}\right) & -\left(\psi_{y z}\right)^{0} \\
-\left(\psi_{z x}\right)^{0} & -\left(\psi_{z y}\right)^{0} & \left(\lambda^{2}-\left(\psi_{z z}\right)^{0}\right)
\end{array}\right|=0
$$

i.e.,

$$
\lambda^{6}+a_{1} \lambda^{4}+a_{2} \lambda^{2}+a_{3}=0
$$

where,

$$
\begin{aligned}
& a_{1}=4-\left(\psi_{x x}\right)^{0}-\left(\psi_{y y}\right)^{0}-\left(\psi_{z z}\right)^{0}, \\
& a_{2}=-\left(\psi_{x y}^{2}\right)^{0}-\left(\psi_{x z}^{2}\right)^{0}-\left(\psi_{y z}^{2}\right)^{0}-4\left(\psi_{z z}^{2}\right)^{0}+\left(\psi_{x x}\right)^{0}\left(\psi_{y y}\right)^{0}+\left(\psi_{x x}\right)^{0}\left(\psi_{z z}\right)^{0}+\left(\psi_{y y}\right)^{0}\left(\psi_{z z}\right)^{0}, \\
& a_{3}=\left(\psi_{x z}^{2}\right)^{0}\left(\psi_{y y}\right)^{0}-2\left(\psi_{x y}\right)^{0}\left(\psi_{x z}\right)^{0}\left(\psi_{y z}\right)^{0}+\left(\psi_{x x}\right)^{0}\left(\psi_{y z}^{2}\right)^{0}+\left(\psi_{x y}^{2}\right)^{0}\left(\psi_{z z}\right)^{0}-\left(\psi_{x x}\right)^{0}\left(\psi_{y y}\right)^{0}\left(\psi_{z z}\right)^{0} .
\end{aligned}
$$

Equation (15) is the characteristic polynomial and we have numerically found the six values of characteristic roots given in tables. In these values at least one is positive root or having positive real part of complex roots (Dark black in the tables). Hence, all the equilibrium points are unstable in all the planes. 
Journal of Nepal Mathematical Society (JNMS), Vol. 2, Issue 1 (2019); A. A. Ansari, P. Kumar, M. Alam

\begin{tabular}{|c|c|c|}
\hline Radiation factor $\beta$ & Equilibria & Corresponding Characteristic Roots \\
\hline \multirow[t]{4}{*}{0.5} & $1.364,0.01$ & $\begin{aligned} \mathbf{2 . 3 5 7 2 1 3 3 2 ,} & -1.90974176 \mathrm{i}, 1.90974176 \mathrm{i},-2.35721332, \\
& -1.97720535 \mathrm{i}, 1.97720535 \mathrm{i}\end{aligned}$ \\
\hline & $-0.0109,-0.0185$ & $\begin{array}{c}-0.49941429-0.99999692 \mathrm{i},-0.49941429+0.99999692 \mathrm{i}, \\
\mathbf{0 . 4 9 9 4 1 4 2 9}+0.99999692 \mathrm{i}, \mathbf{0 . 4 9 9 4 1 4 2 9}-0.99999692 \mathrm{i}, \\
-0.70628673 \mathrm{i}, 0.70628673 \mathrm{i} .\end{array}$ \\
\hline & $-0.67,1.182$ & $\begin{aligned}-2.42607259,- & 1.95211201 \text { i, } 1.95211201 \text { i, }-2.01868444 \text { i, } \\
& 2.01868444 \text { i, } \mathbf{2 . 4 2 6 0 7 2 5 9 .}\end{aligned}$ \\
\hline & $-0.67,-1.1582$ & $\begin{aligned}-2.47287855,- & 1.98117884 \text { i, } 1.98117884 \text { i, }-2.04693700 \mathrm{i}, \\
& 2.04693700 \text { i, } \mathbf{2 . 4 7 2 8 7 8 5 5} .\end{aligned}$ \\
\hline \multirow[t]{4}{*}{0.8} & $1.454,0.001$ & $\begin{aligned}-2.07603417,- & 1.74172796 \text { i, } 1.74172796 \text { i, - } 1.81005567 \text { i, } \\
& 1.81005567 \text { i, } \mathbf{2 . 0 7 6 0 3 4 1 7 . ~}\end{aligned}$ \\
\hline & $-0.0109,-0.0195$ & $\begin{array}{c}-0.63168818-0.99998827 \mathrm{i},-0.63168818+0.99998827 \mathrm{i}, \\
\mathbf{0 . 6 3 1 6 8 8 1 8}+0.99998827 \mathrm{i}, \mathbf{0 . 6 3 1 6 8 8 1 8}-0.99998827 \mathrm{i}, \\
-0.89336770 \mathrm{i}, 0.89336770 \mathrm{i} .\end{array}$ \\
\hline & $-0.72,1.267$ & $\begin{aligned}-2.04385981,-1.72284988 \text { i, } 1.72284988 \text { i, - } 1.79141038 \text { i, } \\
1.79141038 \text { i, } \mathbf{2 . 0 4 3 8 5 9 8 1 .}\end{aligned}$ \\
\hline & $-0.72,-1.242$ & $\begin{array}{c}-2.04040081,-1.72107142 \text { i, } 1.72107142 \text { i, - } 1.78916186 \text { i, } \\
1.78916186 \text { i, 2.04040081. }\end{array}$ \\
\hline \multirow[t]{5}{*}{0.9} & $1.454,0.001$ & $\begin{array}{c}-2.25221769,-1.84738147 \text { i, } 1.84738147 \text { i, }-1.91302538 \text { i, } \\
1.91302538 \text { i, 2.25221769. }\end{array}$ \\
\hline & $-0.0109,-0.0195$ & $\begin{array}{c}-0.67000480-0.99998566 \mathrm{i},-0.67000480+0.99998566 \mathrm{i}, \\
\mathbf{0 . 6 7 0 0 0 4 8 0}+0.99998566 \mathrm{i}, \mathbf{0 . 6 7 0 0 0 4 8 0}-0.99998566 \mathrm{i}, \\
-0.94755953 \mathrm{i}, 0.94755953 \mathrm{i} .\end{array}$ \\
\hline & $-0.732,1.28367$ & $\begin{array}{c}-2.02696867,-1.71332677 \text { i, } 1.71332677 \text { i, - } 1.78132347 \text { i, } \\
1.78132347 \text { i, 2.02696867. }\end{array}$ \\
\hline & $-0.712,-1.2682$ & $\begin{array}{c}-2.02753039,-1.71398738 \text { i, } 1.71398738 \text { i, }-1.78131488 \text { i, } \\
1.78131488 \text { i, } \mathbf{2 . 0 2 7 5 3 0 3 9 .}\end{array}$ \\
\hline & $0.51,0.91$ & $\begin{aligned}-7.62240806,-5.41881682 \text { i, } 5.41881682 \text { i, }-5.46177308 \text { i, } \\
5.46177308 \text { i, } \mathbf{7 . 6 2 2 4 0 8 0 6 .}\end{aligned}$ \\
\hline \multirow[t]{5}{*}{1.0} & $1.514,0.001$ & $\begin{aligned}-1.86697631,- & 1.62146153 \mathrm{i}, 1.62146153 \mathrm{i},-1.69010738 \mathrm{i}, \\
& 1.69010738 \mathrm{i}, \mathbf{1 . 8 6 6 9 7 6 3 1 .}\end{aligned}$ \\
\hline & $-0.0109,-0.0195$ & $\begin{array}{c}-0.70624540-0.99998289 \mathrm{i},-0.70624540+0.99998289 \mathrm{i}, \\
\mathbf{0 . 7 0 6 2 4 5 4 0}-0.99998289 \mathrm{i}, \mathbf{0 . 7 0 6 2 4 5 4 0}+0.99998289 \mathrm{i}, \\
-0.99881545 \mathrm{i}, 0.99881545 \mathrm{i} .\end{array}$ \\
\hline & $-0.732,1.29367$ & $\begin{array}{c}-2.10256574,-1.75820925 \text { i, } 1.75820925 \mathrm{i},-1.82468704 \mathrm{i}, \\
1.82468704 \text { i, 2.10256574. }\end{array}$ \\
\hline & $-0.732,-1.2682$ & $\begin{aligned}-2.09086345,-1.75158070 \text { i, } 1.75158070 \text { i, }-1.81758933 \text { i, } \\
1.81758933 \text { i, } \mathbf{2 . 0 9 0 8 6 3 4 5 .}\end{aligned}$ \\
\hline & $0.51,0.91$ & $\begin{aligned}-7.62240806,- & 5.41881682 \text { i, } 5.41881682 \text { i, }-5.46177308 \text { i, } \\
& 5.46177308 \text { i, } \mathbf{7 . 6 2 2 4 0 8 0 6 .}\end{aligned}$ \\
\hline
\end{tabular}

Table 1: Corresponding characteristic roots of equilibria for the different values of radiation factor $\beta$ in $\mathrm{x}-\mathrm{y}$-plane. 


\begin{tabular}{|c|c|c|}
\hline Radiation factor $\beta$ & Equilibria & Corresponding Characteristic Roots \\
\hline \multirow[t]{2}{*}{0.5} & $1.367,0$ & $\begin{array}{c}-2.32112664,-1.88762721 \text { i, } 1.88762721 \text { i, }-1.95563093 \mathrm{i}, \\
1.95563093 \text { i, } \mathbf{2 . 3 2 1 1 2 6 6 4 .}\end{array}$ \\
\hline & $-0.0109,0$ & $\begin{array}{c}-0.49967858-0.99991052 \text { i, }-0.49967858+0.99991052 \mathrm{i}, \\
\mathbf{0 . 4 9 9 6 7 8 5 8}-0.99991052 \mathrm{i}, \mathbf{0 . 4 9 9 6 7 8 5 8}+0.99991052 \mathrm{i}, \\
-0.70690500 \mathrm{i}, 0.70690500 \mathrm{i} .\end{array}$ \\
\hline \multirow[t]{2}{*}{0.8} & $1.455,0$ & $\begin{aligned}-2.06645094,- & 1.73607684 \text { i, } 1.73607684 \text { i, }-1.80451007 \text { i, } \\
& 1.80451007 \text { i, 2.06645094. }\end{aligned}$ \\
\hline & $-0.0109,0$ & $\begin{array}{c}-0.63195182-0.99979542 \text { i, }-0.63195182+0.99979542 \mathrm{i}, \\
\mathbf{0 . 6 3 1 9 5 1 8 2}-0.99979542 \mathrm{i}, \mathbf{0 . 6 3 1 9 5 1 8 2}+0.99979542 \mathrm{i}, \\
-0.89417196 \mathrm{i}, 0.89417196 \mathrm{i} .\end{array}$ \\
\hline \multirow[t]{2}{*}{0.9} & $1.48,0$ & $\begin{aligned}-2.00679402,- & 1.70150904 \text { i, } 1.70150904 \text { i, - } 1.76977093 \text { i, } \\
& 1.76977093 \text { i, 2.00679402. }\end{aligned}$ \\
\hline & $-0.0109,0$ & $\begin{array}{c}-0.67025651-0.99974999 \text { i, }-0.67025651+0.99974999 \mathrm{i}, \\
\mathbf{0 . 6 7 0 2 5 6 5 1}-0.99974999 \mathrm{i}, \mathbf{0 . 6 7 0 2 5 6 5 1}+0.99974999 \mathrm{i}, \\
-0.94841258 \mathrm{i}, 0.94841258 \mathrm{i} .\end{array}$ \\
\hline \multirow[t]{2}{*}{1} & $1.503,0$ & $\begin{array}{c}-1.95867320,-1.67394958 \text { i, } 1.67394958 \mathrm{i},-1.74192233 \mathrm{i}, \\
1.74192233 \mathrm{i}, \mathbf{1 . 9 5 8 6 7 3 2 0 .}\end{array}$ \\
\hline & $-0.0109,0$ & $\begin{array}{c}-0.70648325-0.99970161 \mathrm{i},-0.70648325+0.99970161 \mathrm{i}, \\
\mathbf{0 . 7 0 6 4 8 3 2 5}-0.99970161 \mathrm{i}, \mathbf{0 . 7 0 6 4 8 3 2 5}+0.99970161 \mathrm{i}, \\
-0.99971464 \mathrm{i}, 0.99971464 \mathrm{i} .\end{array}$ \\
\hline
\end{tabular}

Table 2: Corresponding characteristic roots of equilibria for the different values of radiation factor $\beta$ in $\mathrm{x}$-z-plane.

\begin{tabular}{|c|c|c|}
\hline Radiation factor $\beta$ & Equilibria & Corresponding Characteristic Roots \\
\hline 0.5 & $-0.0181,0$ & $\begin{array}{c}-0.49950884-0.99996864 \mathrm{i},-0.49950884+0.99996864 \mathrm{i}, \\
\mathbf{0 . 4 9 9 5 0 8 8 4}-0.99996864 \mathrm{i}, \mathbf{0 . 4 9 9 5 0 8 8 4}+0.99996864 \mathrm{i}, \\
-0.70650051 \mathrm{i}, 0.70650051 \mathrm{i} .\end{array}$ \\
\hline 0.8 & $-0.0259,0$ & $\begin{array}{c}-0.63158756-0.99985445 \mathrm{i},-0.63158756+0.99985445 \mathrm{i}, \\
\mathbf{0 . 6 3 1 5 8 7 5 6}-0.99985445 \mathrm{i}, \mathbf{0 . 6 3 1 5 8 7 5 6}+0.99985445 \mathrm{i}, \\
-0.89352495 \mathrm{i}, 0.89352495 \mathrm{i} .\end{array}$ \\
\hline 0.9 & $-0.0283,0$ & $\begin{array}{c}-0.66978891-0.99977103 \mathrm{i},-0.66978891+0.99977103 \mathrm{i}, \\
\mathbf{0 . 6 6 9 7 8 8 9 1}-0.99977103 \mathrm{i}, \mathbf{0 . 6 6 9 7 8 8 9 1}+0.99977103 \mathrm{i}, \\
-0.94770723 \mathrm{i}, 0.94770723 \mathrm{i} .\end{array}$ \\
\hline 1 & $-0.0304,0$ & $\begin{array}{c}-0.70589676-0.99966260 \mathrm{i},-0.70589676+0.99966260 \mathrm{i}, \\
\mathbf{0 . 7 0 5 8 9 6 7 6}-0.99966260 \mathrm{i}, \mathbf{0 . 7 0 5 8 9 6 7 6}+0.99966260 \mathrm{i}, \\
-0.99896375 \mathrm{i}, 0.99896375 \mathrm{i} .\end{array}$ \\
\hline
\end{tabular}

Table 3: Corresponding characteristic roots of equilibria for the different values of radiation factor $\beta$ in y-z-plane. 
Journal of Nepal Mathematical Society (JNMS), Vol. 2, Issue 1 (2019); A. A. Ansari, P. Kumar, M. Alam

\section{Conclusion}

In this study, we have performed the behavior of the motion of infinitesimal body in the restricted five-body problem under the assumption that the four primaries are heterogeneous in shapes and sources of radiation pressure. After evaluating the equations of motion, we numerically execute the equilibria, zero-velocity curves and regions of motion in three planes i.e. $x-y, x-z$ and $y-z$ planes, for different values of radiation factor $\beta$. In the $\mathrm{x}-\mathrm{y}$ plane, at $\beta=0.5$ and 0.8 , we got four equilibria but at 0.9 and 1 , we got five equilibria. In the $\mathrm{x}-\mathrm{z}$ plane, we got two equilibria at $\beta=0.9$. And in the $\mathrm{y}-\mathrm{z}$ plane, we got only one equilibrium point at $\beta=0.9$. As far as zero-velocity and regions of motion are concerned, we have plotted these in three planes and explained in detail in the concerning sections. On the other hand, for stability, we have examined it numerically and found that all the equilibria are unstable.

\section{References}

[1] Abdullah, Ahmad, I. and Bhatnagar, K.B., 2009, Periodic orbits of collision in the plane circular problem of four bodies with one of the primaries as an oblate body, Global Sci-Tech, Al-Falah's Journal of Science and Technology, 1(1), Jan-March.

[2] Abouelmagd, E. I. and Mostafa, A., 2015, Out-of-plane equilibrium points locations and forbidden movement regions in the restricted three-body problem with variable mass, Astrophysics Space Sci., 357, 58, DOI 10.1007/s10509-015-2294-7.

[3] Ansari, A. A., 2016, The Photogravitational Circular Restricted Four-body Problem with Variable Masses, Journal of Engineering and Applied Sciences(Majmaah University), 3(2), November.

[4] Ansari, A. A., Kellil, R. and Alhussain, Z., 2017, The effect of perturbations on the circular restricted four-body problem with variable masses, Journal of Mathematics and Computer Science, 17(3), 365377.

[5] Ansari, A. A., Alhussain, Z. and Prasad, S., 2018, The circular restricted three-body problem when both the primaries are heterogeneous spheroid of three layers and infinitesimal body varies it's mass, Journal Of Astrophysics and Astronomy, 39, 57.

[6] Ansari, A. A. and Alhussain, Z., 2018, The restricted five-body problem with cyclic kite configuration, Accepted for publication in Journal of Dynamical Systems and Geometric Theories.

[7] Baltagiannis, A.N. and Papadakis, K.E., 2011, Equilibrium points and their stability in the restricted four-body problem, International Journal of Bifurcation and Chaos, 21, 2179, DOI 10.1142/S0218127411029707.

[8] Bhatnagar, K. B. and Hallan, P. P., 1978, Effect of perturbations in coriolis and centrifugal forces on the stability of equilibrium points in the restricted problem, Celest. Mech. Dyn. Astr., 18, 105-112.

[9] Chernikov, Yu. A., 1970, The photogravitational restricted three-body problem, Soviat Astron A. J., 14,1 .

[10] Kalvouridis, T.J., Arribas, M. and Elipe, A., 2007, Parametric evolution of periodic orbits in the restricted four-body problem with radiation pressure, Planet. Space Sci., 55, 475-493, DOI $10.1016 /$ j.pss.2006.07.005.

[11] Kumari, R. and Kushvah, B. S., 2014, Stability regions of equilibrium points in the restricted four-body problem with oblateness effects, Astrophysics and Space Sci., 349, 693-704.

[12] Marchesin, M. and Vidal, C., 2013, Special restricted rhomboidal five-body problem and horizontal stability of its periodic solutions, Celest. Mech. Dyn. Astro., 115, 261-279, DOI 10.1007/s10569-0129462-7.

[13] Ollongren, A., 1988, On a particular restricted five-body problem: An analysis with computer algebra, J. Symbolic computation, 6, 117-126. 
[14] Papadakis, K.E., 2007, Asymptotic orbits in the restricted four-body problem, Planet. Space Sci., 55, 1368-1379, DOI 10.1016/j.pss.2007.02.005.

[15] Radzievskii, V. V., 1950, The restricted problem of three bodies taking account of light pressure, Akad. Nauk. USSR, Astron. J., 27, 250.

[16] Radzievskii, V. V., 1953, The photo-gravitational restricted three-body problem, Astron. Z., 30(3), 265.

[17] Shoaib, M., Kashif, A.R. and Csillik, I.S., 2017, On the planar central configurations of rohomboidal and triangular four-and five-body problems, Astrophys Space Sci., 362, 182.

[18] Shahbaz, Ullah M., Bhatnagar, K.B. and Hassan, M.R., 2014, Sitnikov problem in the cyclic kite configuration, Astrophys Space Sci., 354, 301-309.

[19] Shalini, K., Ansari, A. A., and Ahmad, F., 2016, Existence and Stability of $L_{4}$ of the $R 3 B P$ When the Smaller Primary is a Heterogeneous Triaxial Rigid Body with N Layers, J. Appl. Environ. Biol. Sci., 6(1), 249-257.

[20] Shalini, K., Suraj, M. S. and Aggarwal, R., 2017, The Non-linear stability of $L_{4}$ in the R3BP when the smaller primary is a Heterogeneous Spheroid, J. of Astronaut Sci., 64(1), 18-49, DOI 10.1007/s40295016-0093-1.

[21] Singh, J. and Leke, O., 2010, Stability of photogravitational restricted three body problem with variable mass, Astrophysics Space Sci., 326 (2), 305-314.

[22] Suraj, M. S., Hassan, M. R. and Asique, M. C., 2014, The Photo-Gravitational R3BP when the Primaries are Heterogeneous Spheroid with Three Layers, J. of Astronaut Sci., 61, 133-155, DOI 10.1007/s40295-014-0026-9.

[23] Suraj, M. S., Aggarwal, R., Shalini, K. and Asique, M. C., 2018, Out-of-Plane Equilibrium points and regions of motion in Photogravitational R3BP when the Primaries are Heterogeneous Spheroid with Three Layers, New Astronomy, 63(8), 15-26.

[24] Zotos, E.E. and Suraj, M.S., 2018, Basins of attraction of equilibrium points in the planar circular restricted five-body problem, Astrophys Space Sci., 363, 20, http:doi.org/10.1007/s10509-017-3240-7. 